\title{
The Impact of Sex and Gender on Adaptation to Space: Executive Summary
}

\author{
Saralyn Mark, MD, ${ }^{1,2,3,4}$ Graham B.I. Scott, PhD, ${ }^{5}$ Dorit B. Donoviel, PhD, ${ }^{6}$ Lauren B. Leveton, PhD, ${ }^{7}$ \\ Erin Mahoney, ${ }^{1,8}$ John B. Charles, $\mathrm{PhD},{ }^{9}$ and Bette Siegel, $\mathrm{PhD}^{1}$
}

\begin{abstract}
This review article is a compendium of six individual manuscripts, a Commentary, and an Executive Summary. This body of work is entitled "The Impact of Sex and Gender on Adaptation to Space" and was developed in response to a recommendation from the 2011 National Academy of Sciences Decadal Survey, "Recapturing a Future for Space Exploration: Life and Physical Sciences for a New Era," which emphasized the need to fully understand sex and gender differences in space. To ensure the health and safety of male and female astronauts during long-duration space missions, it is imperative to examine and understand the influences that sex and gender have on physiological and psychological changes that occur during spaceflight. In this collection of manuscripts, six workgroups investigated and summarized the current body of published and unpublished human and animal research performed to date related to sex- and gender-based differences in the areas of cardiovascular, immunological, sensorimotor, musculoskeletal, reproductive, and behavioral adaptations to human spaceflight. Each workgroup consisted of scientists and clinicians from academia, the National Aeronautics and Space Administration (NASA), and other federal agencies and was co-chaired by one representative from NASA and one from the external scientific community. The workgroups met via telephone and e-mail over 6 months to review literature and data from space- and ground-based studies to identify sex and gender factors affecting crew health. In particular, the Life Sciences Data Archive and the Lifetime Surveillance of Astronaut Health were extensively mined. The groups identified certain sex-related differences that impact the risks and the optimal medical care required by space-faring women and men. It represents innovative research in sex and gender-based biology that impacts those individuals that are at the forefront of space exploration.
\end{abstract}

\section{Introduction and Process}

Q EX AND GENDER SIGNIFICANTLY INFLUENCE health on Earth and in space. To ensure the health and safety of male and female astronauts during long-duration space missions, it is imperative to examine and understand the influences that sex and gender have on physiological and psychological changes that occur during spaceflight.
For over 20 years, there has been an increased effort to comprehend how men and women are similar and different in all aspects of health. Federal agencies have policies to ensure that men and women are included in clinical trials and that findings are evaluated for sex- and gender-based differences. In the 2001 Institute of Medicine report "Does Sex Matter,", "sex" was defined as the classification of male or female according to an individual's genetics and "gender" refers to

\footnotetext{
${ }^{1}$ Advanced Exploration Systems Division, Human Exploration and Operations Mission Directorate, National Aeronautics and Space Administration, Washington, DC.

${ }^{2}$ School of Medicine, Yale University, New Haven, Connecticut

${ }^{3}$ School of Medicine, Georgetown University, Washington, DC.

${ }_{5}^{4}$ DMI, Inc., Bethesda, Maryland.

${ }^{5}$ National Space Biomedical Research Institute, Department of Molecular and Cellular Biology and Center for Space Medicine, Baylor College of Medicine, Houston, Texas.

${ }^{6}$ National Space Biomedical Research Institute, Department of Pharmacology and Center for Space Medicine, Baylor College of Medicine, Houston, Texas.

${ }^{7}$ Behavioral Health and Performance Element, Human Research Program, Division of Biomedical Research and Engineering Science, National Aeronautics and Space Administration, Houston, Texas.

${ }^{8}$ Valador, Inc., Herndon, Virginia.

${ }^{9}$ International Science Office, Human Research Program, National Aeronautics and Space Administration, Houston, Texas.
} 
a person's self-representation as male or female based upon social interactions.

The National Aeronautics and Space Administration (NASA) sponsored a workshop with the University of Missouri in 2002 and published its findings in "Sex, Space and Environmental Adaptation: A National Workshop to Define Research Priorities Regarding Sex-Differences in Human Responses to Challenging Environments."2 The findings included a comprehensive review of existing data and recommendations to fill gaps in NASA's knowledge base.

The 2011 National Academy of Sciences Decadal Survey "Recapturing a Future for Space Exploration: Life and Physical Sciences for a New Era",3 emphasized the need to fully understand sex and gender differences. In response, in 2013, NASA and the National Space Biomedical Research Institute (NSBRI) commissioned a study that resulted in a workshop and this report.

In this study, six workgroups investigated the current body of published and unpublished human and animal research performed to date related to sex- and gender-based differences in the areas of cardiovascular, immunological, sensorimotor, musculoskeletal, reproductive, and behavioral adaptations to human spaceflight. Each workgroup consisted of scientists and clinicians from academia, NASA, and other federal agencies and was co-chaired by one representative from NASA and one from the external scientific community. The workgroups met by telephone and e-mail over 6 months to review literature and data from space- and ground-based studies to identify sex and gender factors affecting crew health. In particular, the Life Sciences Data Archive (LSDA) and the Lifetime Surveillance of Astronaut Health (LSAH) were extensively mined. ${ }^{4,5}$ NASA and NSBRI co-hosted a public virtual workshop on June $25,2013,{ }^{6}$ in which the workgroup co-chairs presented key findings and recommendations for biomedical research priorities.

The remainder of this executive summary provides a synopsis of the key findings and recommendations provided by the six workgroups.

\section{Results and Key Findings}

\section{Key demographics}

As of June 2013, the demographics of the international astronaut and cosmonaut population indicated that a total of 534 humans have flown in space-477 men and 57 women (approximately $11 \%$ of the total). ${ }^{7,8}$ A total of 129 NASA astronauts have flown to the International Space Station (space station), comprising 103 men and 26 women (approximately $20 \%$ of the total). Female NASA space station astronauts are on average 2 years younger than male astronauts. While there were no significant differences in the percentage of male $(76 \%)$ and female space station astronauts $(69 \%)$ who were married, a significantly greater percentage of male astronauts had a least one child $(67 \%$ versus $38 \%$ ) and overall, men had more children than women. From a professional perspective, female NASA space station astronauts have almost twice as many doctorate-level degrees as their male counterparts (50\% versus $28 \%$ ); conversely male NASA space station astronauts had more military experience (73\% versus $39 \%$ ). Sex and gender differences as well as these social determinants could impact adaptation to spaceflight.

\section{Cardiovascular}

On Earth, cardiovascular (CV) disease is the leading cause of death in women and men, with women developing coronary heart disease about a decade later than their male peers. ${ }^{9}$ Although sex and gender gaps continue to narrow, women continue to have greater cardiovascular morbidity and mortality, in part because they do not consistently receive optimal preventive strategies, diagnostic procedures, and treatments. ${ }^{10-12}$ Differences in CV disease on Earth warrant examination of these issues in space.

While there has been a paucity of sex- and gender-based research in this area in space, there have been several notable findings during the past decade (Fig. 1). One important finding is that women experience immediate post-flight orthostatic intolerance, which is the inability to stand without fainting for protracted periods. This condition is more prevalent in female astronauts compared to their male counterparts. ${ }^{13-16}$ A possible mechanism for this sex-based difference is that women have reduced leg vascular resistance as shown during bed rest, which is used as an analog for microgravity. ${ }^{16}$ Additionally, women have greater loss of plasma volume than men during spaceflight. ${ }^{16}$ There are other known sex differences affecting the cardiovascular system. For example, in response to stress, women characteristically respond with an increase in heart rate and men respond with an increase in vascular resistance. ${ }^{17}$ Space implications of these Earth observations require further study.

The visual impairment intracranial pressure (VIIP) syndrome is currently one of the most serious spaceflight-related health risks. ${ }^{18}$ VIIP manifests with anatomical ocular changes, ranging from mild (e.g., globe flattening) to clinically significant (e.g., optic disc edema) symptoms, with a range of corresponding changes in visual function (e.g., hyperopic shifts to enlarged blind spots). In two affected crew members, post-flight lumbar punctures indicated elevated intracranial pressure (ICP) (i.e., $>25 \mathrm{~cm} \mathrm{H}_{2} \mathrm{O}$ ). Prolonged elevation of ICP can cause long-term loss of visual acuity and potentially also impact neurological function. ${ }^{19}$

NASA has a newly developed research program aimed at identifying the underlying pathophysiology of VIIP, which would then allow for countermeasure development. Currently, $82 \%$ of male astronauts are affected (14 out of 17 studied) versus $62 \%$ of female astronauts (5 out of 8 studied). This difference is not statistically significant, potentially due to the small number of subjects (particularly female astronauts) studied to date. All clinically significant cases described thus far have occurred in male astronauts, ${ }^{20,21}$ while female astronauts have exhibited much milder visual impairment symptoms. The observation that clinically significant cases of visual impairment have occurred only in male astronauts may be related to several factors including higher vascular compliance in women, which could be protective, and the slightly younger age of female astronauts compared with male astronauts. Sex hormone-related differences could impact vascular compliance and plasma volume alterations. Research is underway to better understand individual susceptibilities (including sex differences) as well as environmental and behavioral factors associated with the VIIP syndrome.

\section{Immunologic}

Earth-based studies suggest sex differences in immune responses. Women mount a more potent immune response than 

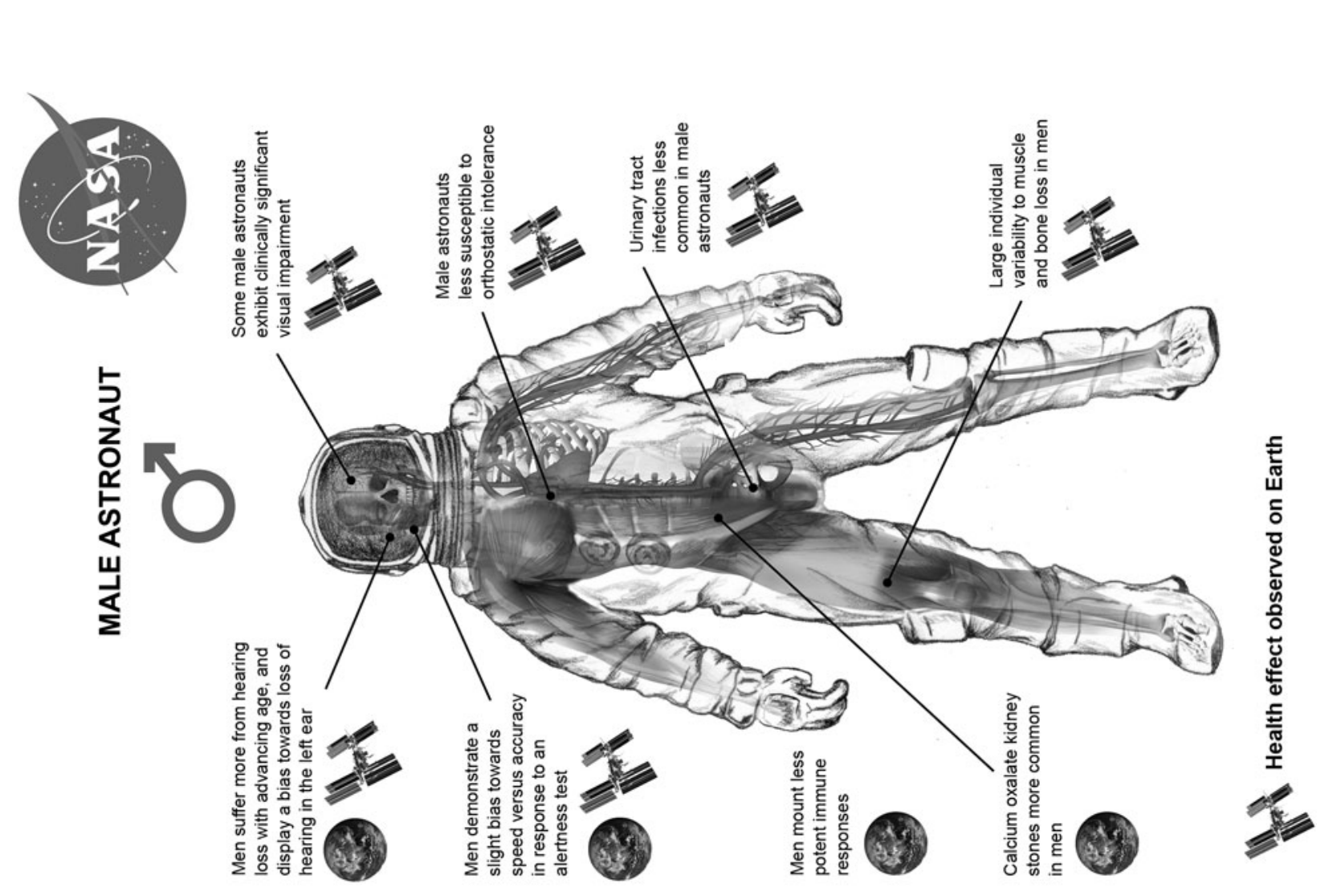

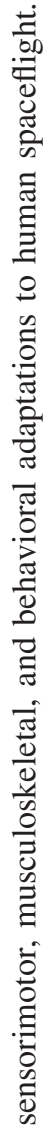
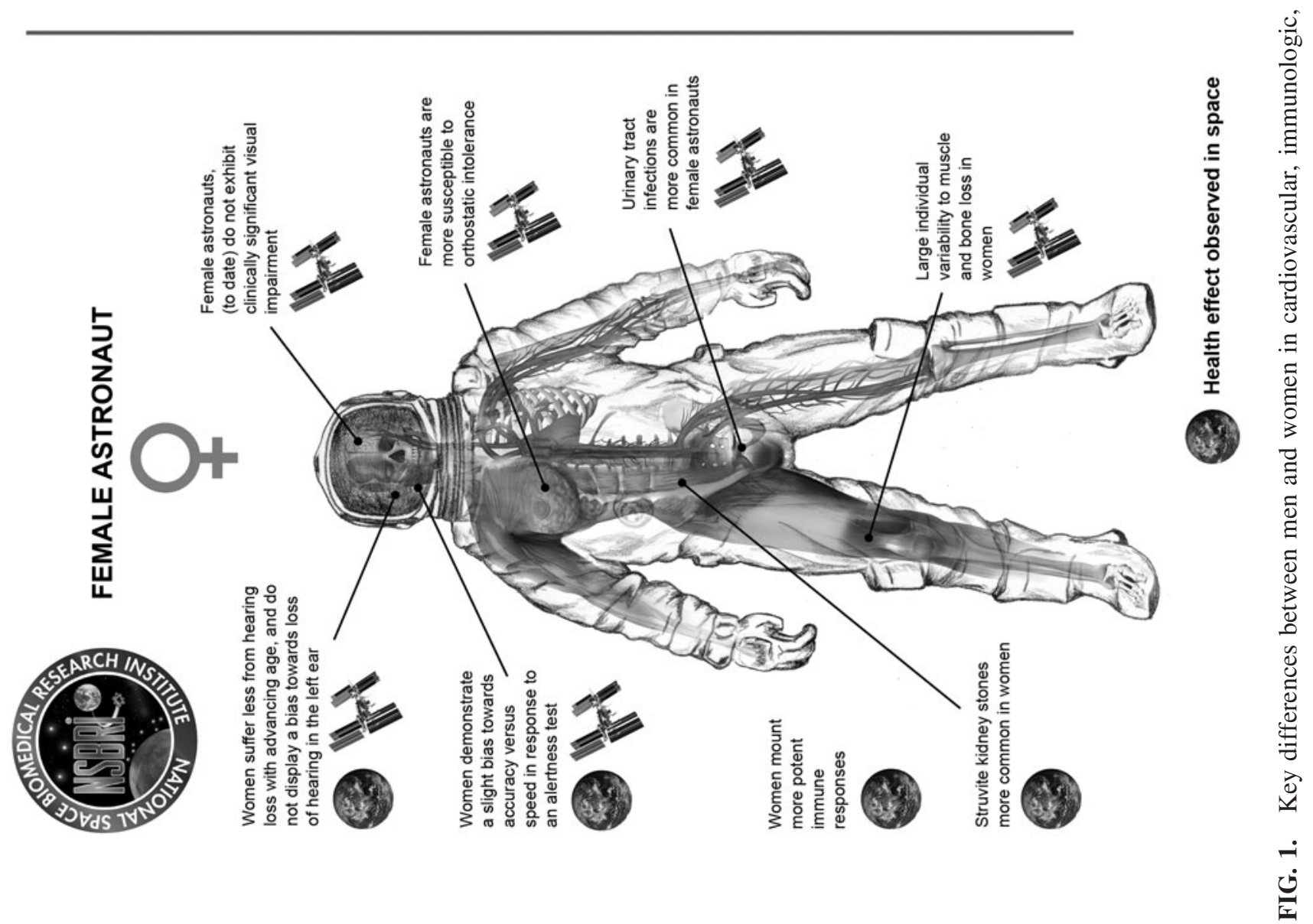
men, including enhanced production of antibody and cellmediated immune responses. ${ }^{22,23}$ This makes them more resistant than men to viral and bacterial infections; and, once infected, women mount more robust responses. As a corollary, an activated immune system results in higher rates of autoimmunity; as such, over $70 \%$ of people affected by autoimmune diseases are women. ${ }^{24,25}$ These differences may be attributed to the impact of sex hormones such as estrogen, progesterone, and testosterone on immune cell function, with each hormone having distinct effects.

Changes in function and concentration of key constituents of the immune system related to spaceflight have been reported. In addition, reactivation of latent viral antigens occurs in flight, which may persist post flight. ${ }^{26-28}$ Accordingly, an exploration mission may increase crew risk for adverse health effects, such as infectious disease, hypersensitivities, autoimmunity, and malignancy.

Radiation represents a major hazard for space travel. Ionizing radiation deleteriously affects hematopoietic and other immune system cells. ${ }^{29,30}$ It has been reported that female subjects are more susceptible to radiation-induced cancer than are male subjects. ${ }^{31}$ In humans, radiation-induced breast cancer contributes to this increased risk for women. ${ }^{31}$ Moreover, there is also an increased risk for other major types of radiation-induced cancer, such as lung cancer, in women compared with men. ${ }^{31}$

\section{Sensorimotor}

Men and women differ in most sensory systems, neural anatomy, and functional responses. Anatomical sex differences exist within the human brain, including a more active corpus callosum and a larger hippocampus in women and a larger amygdala in men. The sexes also differ in neuron differentiation and development and in neurochemical pathways. $^{32}$

Sex differences in the visual senses include circular vision (orientation within a spinning environment), field dependence (perceiving orientation based only on visual cues), perception of vertical with body tilt (correctly identifying the true vertical to the ground when the body is titled), and perception of the horizon. ${ }^{33,34}$ These differences may be due to biological differences within the vestibular system, including differences in the inner ear structures, which are significantly larger in males. ${ }^{35}$ Men have greater sensitivity for fine detail and for rapidly moving stimuli, while women exhibit better color discrimination, possibly because many men suffer from genetically inherited color blindness. ${ }^{36,37}$

The sexes respond differently to stress. In rodents, stress enhances performance in males but impairs it in females. ${ }^{38}$ The susceptibility of hippocampal cells to chronic stress has been suggested to play a role in post-traumatic stress disorder and clinical depression, which are disorders that disproportionately affect women. ${ }^{39}$ Moreover, a brief exposure to a stressful learning situation increases the density of dendritic spines in male rats but decreases spine density in female rats. ${ }^{40}$

The common belief is that women are more susceptible to motion sickness on Earth; however, laboratory tests do not robustly support this assertion. Clinically, women on Earth present with more vestibular disorders such as vertigo, possibly due to the fact that they have fewer myelinated axons in the vestibular nerve than men. ${ }^{41-43}$
Upon transition to microgravity on space station missions, female astronauts reported a slightly higher incidence of space motion sickness (SMS) compared with men (i.e., 50\% of women reported experiencing SMS versus $38 \%$ of men). Conversely, during return to Earth, male space station astronauts experience entry motion sickness symptoms more frequently (47\%) than their female counterparts (40\%). These differences are not statistically significant, likely due to the small sample size of the female astronauts within this dataset. These data were compiled via analysis of NASA's LSAH database. ${ }^{5}$

Hearing sensitivity, when measured at most frequencies, declines much more rapidly in male astronauts than it does in female astronauts. These LSAH derived data represent a wide age range of subjects (i.e., four decades) and show a more rapid decline in hearing in the left ear, for men only. Within the general population, hearing also declines more rapidly in men than in women, due in part to environmental factors or occupational exposure (e.g., construction or factory work). No evidence suggests that the sex-based hearing differences in the astronaut population are related to microgravity exposure, and the small sample size of female astronauts precludes making any definitive conclusions. ${ }^{44}$

\section{Musculoskeletal}

The human musculoskeletal response to unloading is highly variable among individuals, with tenfold differences often observed. For example, after 30 days of unilateral lower limb suspension in animals, individual responses in muscle loss ranged from $2.5 \%$ to a nearly $20 \%$ in plantarflexor cross-sectional area compared with before the suspension. ${ }^{45}$ Similarly, 6 months in microgravity aboard the Mir space station resulted in a $2 \%$ to $24 \%$ loss of cancellous bone in the distal tibia. ${ }^{46}$ These individual differences make it difficult to ascertain whether there are sex-specific effects of unloading. The initial start point for bone and muscle may influence the rate of loss with unloading in spaceflight and whether that rate of loss is linear over an approximately 3-year period, which is the approximate timeframe for current notional concepts of Mars surface missions. This is particularly germane when developing countermeasures for women and men, since men generally have greater muscle and bone mass.

On Earth, osteoarthritis of the knee is significantly more common in women than in men. ${ }^{47}$ Sex-based risk factors include the loss of estrogen's anabolic effect on cartilage after menopause, a higher incidence of predisposing knee injuries such as anterior cruciate ligament tears, and increased joint laxity in women. ${ }^{48}$ Since muscles serve to stabilize and dampen forces across joints, ${ }^{49}$ loss of muscle mass and strength after prolonged unloading can contribute to joint injury risk and early degenerative joint changes, especially in the knee.

\section{Reproductive}

There are several reproductive health concerns for astronauts in space that may be primarily related to microgravity and radiation. In both populations, temporary infertility has been associated with high-dose, acute ionizing radiation exposures, as the gonads are highly sensitive to such exposures. ${ }^{50}$ Women have a higher incidence of radiation-induced cancers, largely driven by lung, thyroid, breast and ovarian cancers, and therefore are permitted to spend significantly 
less time in space than men. ${ }^{51}$ For example, a 45-year-old man has a 344-day limit versus a 187-day limit for a 45-yearold woman, due to radiation exposures on the space station that would be typical at the time of solar maximum. Theoretically, there is also more time for post-flight carcinogenesis to occur in women due to their increased longevity.

Thirteen female astronauts have given birth to 18 children following spaceflight and have not experienced any increased pregnancy complications or increased assisted reproductive technology failures compared to the general population (E. S. Baker, unpublished data). There have not been systematic follow-up health studies of the offspring. Human bed rest studies found reductions in live spermatozoa, suggesting that spermatogenesis in men may be affected by exposure to microgravity. ${ }^{52}$

Several hypothalamic transmitters are altered by real or simulated spaceflight. Alterations in hypothalamic pituitary gonadal and hypothalamic pituitary adrenal axes have been reported following spaceflight. Changes include reduced levels of testosterone in men ${ }^{53}$ that appear to rebound upon return to Earth. ${ }^{54}$ Oxytocin dampens the stress response in men and women by reducing cortisol levels. ${ }^{55,56}$ Oral contraceptives (OC) reduce the release of oxytocin as well as cortisol. ${ }^{57}$ Therefore, females using OCs during spaceflight may not experience increased cortisol levels and the associated stress response.

On Earth, calcium oxalate urinary tract stones are more common in Caucasian men, and struvite stones are more commonly observed in Caucasian women. ${ }^{58}$ While no confirmed cases of urinary tract stones have been reported in the U.S. space program during spaceflight, male and female astronauts have experienced stones post flight. There is an increased incidence of spaceflight-associated dehydration and hypercalciuria, thereby increasing the risk of calcium oxalate stones in both female and male astronauts. ${ }^{59}$

While the overall incidence is not much different from that found on Earth, urinary tract infections in space are more common in women and have been successfully treated with antibiotics. ${ }^{60}$ Transient difficulties with urination are reported by both sexes, but the only astronauts requiring catheterization have been women. Possible explanations include differences in hydration, adjustment to voiding in microgravity, and use of antiemetics.

Regarding conception, reptilian eggs have been successfully fertilized during spaceflight; ${ }^{61}$ however, only one mating experiment in mice has been conducted in spaceflight and there were no viable offspring. ${ }^{62}$

\section{Behavioral}

Analysis of space station astronaut neurobehavioral and sleep measures showed no sex or gender differences in alertness using the Psychomotor Vigilance Test. ${ }^{63}$ Similarly, no significant sex differences have been identified thus far using self-ratings of workload, tiredness and stress, or sleep quality in-flight or post-flight (D. F. Dinges, personal communication, 2013).

Ground-based research on sleep and circadian rhythms suggests that men gain significantly more weight than women during chronic sleep restriction. ${ }^{64}$ Furthermore, chronic sleep restriction may induce greater increases in leptin, ${ }^{65}$ as well as greater cellular immune activation of interleukin- 6 and tumor necrosis factor-alpha in women than men. ${ }^{66}$
On Earth, anxiety and major depressive disorders are about twice as common in women than in men. ${ }^{67}$ Symptoms, diagnoses, comorbidities, and responses to treatment differ for men and women. ${ }^{58,68-70}$ There is no evidence that female astronauts experience the same risk for depressive and anxiety disorders as their counterparts in the general population. Because all astronaut candidates undergo a robust process of psychological screening and selection, the likelihood of sex differences in affective disorders may be reduced.

\section{Conclusions}

Informed decision-making regarding risks, countermeasures, and medical treatments for long-duration exploration missions requires a more thorough understanding of sex and gender differences in adaptation. Many questions remain unanswered. For example, to date, the more clinically significant cases of spaceflight-induced visual impairment have been observed only in male astronauts, but the sample size of longduration female astronauts is still relatively modest. Conversely, orthostatic intolerance manifests more frequently in women. There may be individual and sex differencesregarding hormone, stress, and immune responses, the sensory system, and the circadian system-that are important to understand for planning exploration-class missions and designing spacecraft. These factors also influence how astronauts will safely and productively live and work in space.

Interdependencies exist between the six physiological areas that have been discussed in this analysis. The development of appropriate and effective countermeasures will require an integrated approach. Radiation, for instance, represents a harsh element of the space environment meriting more comprehensive research; for this analysis, it was thoroughly reviewed only in the areas of reproduction and immunology.

With regard to the recommendations detailed in the 2002 report, NASA has made some progress. LSAH and LSDA serve as repositories and resources for applied and clinical studies for the research community and were utilized by the six workgroups throughout this investigation. Increasingly, the design and execution of experiments funded by NASA and NSBRI have considered sex and gender in subject selection and data analyses. Inevitably, space research involves small numbers of test subjects. Current NASA and NSBRI research announcements provide guidance regarding the appropriate statistical handling of this special research challenge. In the latest crew selection, NASA selected eight astronauts, comprising four women and four men. This is a positive step toward increasing the participation of female astronauts in spaceflight and experimentation.

This analysis has heavily focused on sex differences because the data currently available have been amenable to such an approach. In the future, sex and gender differences will constitute increasingly important components of an overall personalized medicine approach to protecting the health of humans on Earth and in space.

\section{Recommendations}

- Select more female astronauts for space missions.

- Encourage and facilitate the participation of more female and male subjects in both ground and flight research studies. 
- Focus on the responses of individual astronauts to spaceflight and return to Earth.

- Determine the range of effectiveness of specific countermeasures for individuals.

- Include sex and gender factors into the design of human spaceflight experiments.

- Incorporate sex and gender and other individual risk factors into NASA-funded research programs.

\section{Acknowledgments}

G.B.I.S. and D.B.D. are grateful for the support provided by NSBRI through NASA Cooperative Agreement NCC 958. B.S., E.M., and S.M. are grateful for the support provided by the NASA Advanced Exploration Systems Division. L.B.L. and J.B.C. are grateful for the support provided by the NASA Johnson Space Center Human Research Program and the Division of Biomedical Research and Environmental Sciences Division.

\section{Author Disclosure Statement}

No competing financial interests exist.

\section{References}

1. Institute of Medicine. Exploring the biological contributions to human health: Does sex matter? Washington, DC: National Academies Press, 2001. http://www.iom.edu/ /media/Files/Report\%20 Files/2003/Exploring-the-Biological-Contributions-to-Human-HealthDoes-Sex-Matter/DoesSexMatter8pager.pdf Accessed November 5, 2014

2. National Aeronautics and Space Administration (NASA) and the National Center for Gender Physiology and Environmental Adaptation and University of Missouri. Sex, space and environmental adaptation: A national workshop to define research priorities regarding sex-differences in human responses to challenging environments. Columbia, MO, November 12-14, 2002. Available at www.nasa.gov/pdf/185051main_environmental_adaptation_work shop_11-2002.pdf Accessed May 30, 2014.

3. National Research Council. Recapturing a future for space exploration: Life and physical sciences for a new era. Washington, DC: The National Academies Press, 2011. Available at www.nap.edu/ catalog.php?record_id=13048 Accessed May 30, 2014.

4. National Aeronautics and Space Administration (NASA). Life sciences data archive. Houston, Texas. Available at http://lsda.jsc .nasa.gov/ Accessed May 30, 2014.

5. National Aeronautics and Space Administration (NASA). Lifetime surveillance of astronaut health. Current research. Houston, Texas. Available at http://lsda.jsc.nasa.gov/docs/research/research_detail .aspx? experiment_type_code $=40 \&$ researchtype $=$ current Accessed May 30, 2014.

6. National Aeronautics and Space Administration (NASA) and National Space Biomedical Research Institute (NSBRI). Washington, D.C. The impact of sex \& gender on adaptation to space virtual workshop archive. Available at http://www.nasa.gov/exploration/ library/events/gender-workshop.html Accessed May 30, 2014.

7. Chinatopix. 57 of the 534 people that have flown to space are women. Available at http://www.chinatopix.com/articles/353/20130618/ 57-534-people-flown-space-women.htm Accessed May 30, 2014.

8. Wikipedia. List of female astronauts. Available at http://en.wikipedia .org/wiki/List_of_female_astronauts Accessed May 30, 2014.

9. Hochman JS, Tamis, JE, Thompson TD, et al. Sex, clinical presentation, and outcome in patients with acute coronary syndromes. N Engl J Med 1999;341:226-232.

10. Vaccarino V, Parsons L, Every NR, Barron HV, Krumholz HM. Sex-based differences in early mortality after myocardial infarc- tion. National Registry of Myocardial Infarction 2 Participants. N Engl J Med 1999 22;341217-1225.

11. Jacobs AK. Contemporary reviews in interventional cardiology; coronary intervention in 2009: Are women no different than men? Circ Cardiovasc Intervent 2009;2:69-78. Available at http:// circinterventions.ahajournals.org/content/2/1/69.full?sid=074e1fe 7 d91a-4e98-a0eb-745a62167954 Accessed May 30, 2014.

12. Wenger NK. Women in cardiology: Are we there yet? Closing the gender gap in coronary heard disease recognition, management and outcomes. Expert Rev Cardiovasc Ther. 2013;11:1447-1450.

13. Blaber AP, Bondar RL, Kassam MS. Heart rate variability and short duration spaceflight: relationship to post-flight orthostatic intolerance. BMC Physiol 2004;4:6.

14. Blaber AP, Goswami N, Bondar RL, Kassam MS. Impairment of cerebral blood flow regulation in astronauts with orthostatic intolerance after flight. Stroke 2011;42:1844-1850.

15. Cooper G, Kent RL, Mann DL. Load induction of cardiac hypertrophy. J Mol Cell Cardiol 1989;21 Suppl 5:11-30.

16. Waters WW, Ziegler MG, Meck JV. Postspaceflight orthostatic hypotension occurs mostly in women and is predicted by low vascular resistance. J Appl Physiol 2002;92:586-594.

17. Martin EA, Tan SL, MacBride LR, Lavi S, Lerman LO, Lerman A. Sex differences in vascular and endothelial responses to acute mental stress. Clin Auton Res 2008;18:339-345.

18. National Aeronautics and Space Administration (NASA). The visual impairment intracranial pressure summit report, October 2011. Houston, Texas. Available at http://ston.jsc.nasa.gov/collections/ trs/_techrep/TP-2011-216160.pdf Accessed May 30, 2014.

19. Friedman DI. Idiopathic intracranial hypertension. Curr Pain Headache Rep 2007;11:62-68.

20. Mader TH, Gibson CR, Pass AF, Kramer LA, Lee AG et al. Optic disc edema, globe flattening, choroidal folds, and hyperopic shifts observed in astronauts after long-duration space flight. Ophthalmology 2011;118:2058-2069.

21. Kramer LA, Sargsyan AE, Hasan KM, Polk JD, Hamilton DR. Orbital and intracranial effects of microgravity: Findings at 3-T MR imaging. Radiology 2012;263:819-827.

22. Spencer MJ, Cherry JD, Powell KR, et al. Antibody responses following Rubella immunization analyzed by HLA and ABO types. Immunogenetics 1977;4:365-372.

23. Goble FC, Konopka EA. Sex as a factor in infectious diseases. Trans NY Acad Sci 1973;35:325-346.

24. Whitacre, CC. Sex differences in autoimmune disease. Nat Immunol 2001:777-780.

25. Beeson PB. Age and sex associations of 40 autoimmune diseases. Am J Med 1994;96:457-462.

26. Pierson D, Mehta S, Bruce R, Ott CM. Role of environmental factors in immunity and infectious disease risk. Presented at 35th International Conference on Environmental Systems, Rome, Italy, 2005

27. Cohrs RJ, Mehta SK, Schmid DS, Gilden DH, Pierson DL. Asymptomatic reactivation and shed of infectious varicella zoster virus in astronauts. J Med Virol 2008;80:1116-1122.

28. Mehta SK, Stowe RP, Feiveson AH, Tyring SK, Pierson DL. Reactivation and shedding of cytomegalovirus in astronauts during spaceflight. J Infect Dis 2000;182:1761-1764.

29. Sanzari JK, Wan XS, Wroe AJ, et al. Acute hematological effects of solar particle event proton radiation in the porcine model. Radiat Res 2013;180:7-16.

30. Gridley DS, Freeman TL, Makinde AY, et al. Comparison of proton and electron radiation effects on biological responses in liver, spleen and blood. Int J Radiat Biol 2011;87:1173-1181.

31. Committee on the Biological Effects of Ionizing Radiation (BEIR VII). Health effects of exposure to low levels of ionizing radiation. Washington, DC: National Academy of Sciences, National Research Council, 2006

32. Cahill L. Why sex matters for neuroscience. Nat Rev Neurosci 2006;7:477-484.

33. Darlington CL, Smith PF. Further evidence for gender differences in circularvection. J Vestib Res 1998;8:151-153. 
34. Tremblay L, Elliott D. Sex differences in judging selforientation: the morphological horizon and body pitch. BMC Neurosci 2007;8:6.

35. Sato H, Sando I, Takahashi H. Computer-aided three-dimensional measurement of the human vestibular apparatus. Otolaryngol Head Neck Surg 1992;107:405-409.

36. Abramov I, Gordon J, Feldman O, Chavarga A. Sex and vision II: Color appearance of monochromatic lights. Biol Sex Differ 2012; $3: 21$.

37. Abramov I, Gordon J, Feldman O, Chavarga A. Sex and vision I: Spatio-temporal resolution. Biol Sex Differ 2012;3:20.

38. Shors, T. Opposite effects of stressful experience on memory function in males versus females. Dialogs Clin Neurosci 2002;4: 139-147.

39. Sherin JE, Nemeroff CB. Post-traumatic stress disorder: The neurobiological impact of psychological trauma. Dialogues Clin Neurosci 2011;13:263-278.

40. Shors TJ., Falduto J, Leuner B. The opposite effects of stress on dendritic spines in male vs. female rats are NMDA receptordependent. Eur J Neurosci 2004;19:145-150.

41. Moriyama H, Itoh M, Shimada K, Otsuka N. Morphometric analysis of fibers of the human vestibular nerve: Sex differences. Eur Arch Otorhinolaryngol 2007;264:471-475.

42. Neuhauser HK, von Brevern M, Radtke A, et al. Epidemiology of vestibular vertigo: a neurotologic survey of the general population. Neurology 2005;65:898-904.

43. von Brevern M, Radtke A, Lezius F, et al. Epidemiology of benign paroxysmal positional vertigo: a population based study. J Neurol Neurosurg Psychiatry 2007;78:710-715.

44. Marlenga B, Berg RL, Linneman JG, Wood DJ, Kirkhorn SR, Pickett W. Determinants of early-stage hearing loss among a cohort of young workers with 16-year follow-up. National Center for Biotechnology Information. PubMed Central, March 2012. Available at www.ncbi.nlm.nih.gov/m/pubmed/22447644/?i=16\&from= hearing\%20loss\%20men\%20women\%20prevalence Accessed May 30, 2014.

45. Clark BC, Fernhall B, Ploutz-Snyder LL. Adaptations in human neuromuscular function following prolonged unweighting: I. Skeletal muscle contractile properties and applied ischemia efficacy. J Appl Physiol 2006;101:256-263.

46. Vico L, Collet P, Guignandon A, et al. Effects of long-term microgravity exposure on cancellous and cortical weight-bearing bones of cosmonauts. Lancet 2000;355:1607-1611.

47. Boyan BD, Hart DA, Nicolella DP et al. Hormonal modulation of connective tissue homeostasis and sex differences in risk for osteoarthritis of the knee. Biol Sex Differ 2013;4:6410-6414.

48. Myer G, Ford K, Paterno M, Nick T, Hewett T. The effects of generalized joint laxity on risk of anterior cruciate ligament injury in young female athletes. Am J Sports Med, March 2008; PubMed Central, July 2012. Available at http://www.ncbi.nlm.nih.gov/ pmc/articles/PMC3407802/ Accessed May 30, 2014.

49. Hudelmaier M, Glaser C, Englmeier KH, Reiser M, Putz R, Eckstein F. Correlation of knee-joint cartilage morphology with muscle crosssectional areas vs. anthropometric variables. Anat Rec A Discov Mol Cell Evol Biol 2003;270:175-184.

50. Rubin P. Law and order of radiation sensitivity: absolute versus relative. In: Vaeth JM, Meyer JL, eds. Frontiers of radiation therapy and oncology. Basel: Karger, 1989:7-40.

51. Cucinotta FA, Kim MA, Chappell L. Space radiation cancer risk projections and uncertainties- 2012. NASA TP 2013-217375, 2013.

52. Nichiporuk IA, Evdokimov VV, Erasova VI, et al. Male reproductive system in conditions of bed-rest in a head-down tilt. J Gravit Physiol 1998;5:101-102.

53. Strollo F, Riondino G, Harris B, et al. The effect of microgravity on testicular androgen secretion. Aviat Space Environ Med 1998; 69:133-136.
54. Ortiz RM, Wade CE, Morey-Holton E. Urinary excretion of LH and testosterone from male rats during exposure to increased gravity: Post-spaceflight and centrifugation. Proc Soc Exp Biol Med 2000;225:98-102.

55. Legros JJ, Chiodera P, Geenen V, Smitz S, Von Frenckell R. Doseresponse relationship between plasma oxytocin and cortisol and adrenocorticotropin concentrations during oxytocin infusion in normal men. The Endocrine Society, July 2013. Available at http:// press.endocrine.org/doi/abs/10.1210/jcem-58-1-105 Accessed May 30, 2014

56. Heinrichs M, Baumgartner T, Kirschbaum C, Ehlert U. Social support and oxytocin interact to suppress cortisol and subjective responses to psychosocial stress. Biol Psychiatry 2003;54:1389-1398.

57. Salonia A, Nappi RE, Pontillo M, et al. Menstrual cycle-related changes in plasma oxytocin are relevant to normal sexual function in healthy women. Horm Behav 2005;47:164-169.

58. Dall'Era J, Kim F, Chandhoke S. Gender differences among Hispanics and Caucasians in symptomatic presentation of kidney and ureteral stones. J Endourol 2005;19:283.

59. Liakopoulos V, Leivaditis K, Eleftheriadis T et al. The kidney in space. Int Urol Nephrol 2012 44:1893-1901.

60. Jennings RT, Baker ES. Gynecologic and reproductive concerns. In: Barratt, MR and Pool, SL (eds). Principles of clinical medicine for space flight. Springer, in review.

61. Aimar C, Bautz A, Durand D, et al. Microgravity and hypergravity effects on fertilization of the salamander Pleurodeles waltl (urodele amphibian). Biol Reprod 2000;63:551-558.

62. Keefe JR. Final report of the NASA Mammalian Developmental Biology Working group. In: Souza KA, Halstead TW, eds. NASA developmental biology workshop. NASA TM-86756, Washington, DC 1985:46-63.

63. Basner M, Mollicone D, Dinges DF. Validity and sensitivity of a Brief Psychomotor Vigilance Test (PVT-B) to total and partial sleep deprivation. Acta Astronaut 2011;69:949-959.

64. Spaeth AM, Dinges DF, Goel N. Effects of sleep restriction on weight gain, caloric intake and meal timing in healthy adults. Sleep 2013;36:981-990.

65. Simpson NS, Banks S, Dinges DF. Sleep restriction is associated with increased morning plasma leptin concentrations, especially in women. Biol Res Nurs 2010;12:47-53.

66. Irwin MR, Carrillo C, Olmstead R. Sleep loss activates cellular markers of inflammation: Sex differences. Brain Behav Immun 2010;24:54-57.

67. American Psychiatric Association. Diagnostic and statistical manual of mental disorders, 5th ed. Washington, DC: American Psychiatric Association, 2013.

68. Kornstein SG, Sloan DM, Thase ME. Gender-specific differences in depression and response to antidepressant treatment. Psychopharmacol Bull 2002;36:99-112.

69. Marcus SM, Kerber KB, Rush AJ, et al. Gender differences in depression symptoms in treatment-seeking adults: Confirmatory analyses from the Sequenced Treatment Alternatives to Relieve Depression study. Compr Psychiatry 2008;49:238-236.

70. Pigott TA. Anxiety disorders in women. Psychiatr Clin North Am 2003;26:621-672.

Address correspondence to: Bette Siegel, PhD

Human Exploration and Operations Mission Directorate National Aeronautics and Space Administration 300 E Street Southwest Washington, DC 20546

E-mail: bette.siegel@nasa.gov 